\title{
Identification of osteosarcoma driver genes using a network method
}

\author{
ZEBING SI and KONGHE HU \\ Department of Orthopedics, The Affiliated Yuebei People's Hospital of Shantou University Medical College, \\ Wujiang, Shaoguan 512026, P.R. China
}

Received March 12, 2019; Accepted November 7, 2019

DOI: $10.3892 / \mathrm{ol} .2019 .11212$

\begin{abstract}
Osteosarcoma (OS) is a severe disease that is generally caused by genetic alterations. Systematic identification of driver genes may be used to increase the understanding of the mechanisms underlying the disease. The present study identified a framework to predict driver genes, with the hypothesis that driver genes operate through a number of connected functional genes. OS-related genes were extracted from the Catalogue Of Somatic Mutations In Cancer and subsequently ranked by virtue of their effect on a set of functional genes using a network-based algorithm. This revealed the driver genes associated with dysregulated networks. In addition, compared with the Mutations For Functional Impact on Network Neighbors algorithm, the results obtained using the aforementioned network-based algorithm revealed that the proposed method is effective. Gene functional analysis demonstrated that the potential OS driver genes were involved in OS-associated pathways. Through the validation of the 15 candidate OS driver genes, the classifier constructed in the present study revealed that the identified driver genes were able to distinguish 184 cancer samples from controls. Therefore, the present study provided insights into the identification of driver genes from a vast amount of sequencing data.
\end{abstract}

\section{Introduction}

Osteosarcoma (OS) is a malignant bone tumor that often occurs in children and adolescents (1). Improving the 5-year survival rate remains a challenge (2). OS is characterized by the accumulation of somatic mutations, including gross insertions and deletions (3). With the development of next generation sequencing, an increasing number of OS-associated

Correspondence to: Dr Konghe Hu, Department of Orthopedics, The Affiliated Yuebei People's Hospital of Shantou University Medical College, 133 Shaoguan Huimin South Avenue, Wujiang, Shaoguan 512026, P.R. China

E-mail: hkhgksq@126.com

Key words: osteosarcoma, gene, network, catalogue of somatic mutations in cancer mutations have been identified. However, only a small proportion of these represent driver mutations, as the majority are passenger mutations (4). The identification of driver mutations may improve the understanding of the molecular mechanisms underlying OS, as well as provide potential diagnostic and therapeutic markers. Therefore, the development of accurate automated computational prediction algorithms capable of screening driver from passenger mutations is of paramount importance.

The development of next-generation sequencing technology has allowed the production of a vast amount of mutation data, which in turn stimulated the development of algorithms for the identification of variants that are likely to be associated with disease (5). The Catalogue of Somatic Mutations in Cancer (COSMIC) is a comprehensive resource for cataloguing somatic mutations in human tumors (6). However, biological experiments that investigate the effect of each gene/mutation are time-consuming and not cost-effective. Computational methods, on the other hand, are able to mine vast datasets for mutation information. A case group was constructed using pathogenic mutations (melanoma-associated mutations) identified using COSMIC (7). All point mutations in COSMIC can be classified as pathogenic or neutral variants using the algorithm Functional Analysis Through Hidden Markov Models (FATHMM)-Math Kernel Library (MKL) (8). FATHMM is highly precise, with only a small proportion of false positive somatic mutations (8), and is widely used to filter variants and to detect driver genes (9). However, as FATHMM is not a cancer-specific prediction tool, improving the accuracy of predicting driver genes for a specific type of cancer is urgently required. Furthermore, cancer development is generally a result of mutations in multiple genes as opposed to a single gene. Therefore, network-based methods that consider the interaction between genes may be advantageous.

While the detection of driver network modules implicates the constituent genes as being cancer-associated, several methods have been developed to directly identify genes involved in cancer pathogenesis (5). Direct implication of genes may reduce false positive driver gene prediction in cases where not all genes in a network module have equal oncogenic potential. Although many gene-level methods rely on patterns of mutation, networks have also been applied to implicate driver genes. Mutations For Functional Impact on Network Neighbors (MUFFINN) is a pathway-centric method 
that identifies cancer-associated genes based on the mutation data of both individual genes and their neighbors connected in functional networks (10). Application of MUFFINN revealed that analysis of mutations in indirect neighbors via diffusion algorithms did not improve the predictive performance compared to analysis of only direct neighbors in 18 types of cancer (10).

The present study performed a systematic exploration of somatic mutations by mining datasets for OS-associated driver genes using a network-based approach. Firstly, the mutation impact scores calculated by FATHMM based on COSMIC were integrated, and only the pathogenic mutations were selected for further study. Secondly, as the power to detect driver genes depended on how many mutated genes were connected with functional genes, a protein-protein interaction (PPI) network consisting of mutated and functional genes was created. Subsequently, the following method was used to uncover the driver genes that were associated with the functional genes. For each mutated gene, the enrichment score for known functional genes was calculated using a network approach, and the number of driver genes was summarized into a driver-gene score to evaluate the function of the driver genes. Furthermore, the identified driver genes were validated using an independent validation dataset. The results revealed that the driver genes may be used as biomarkers to predict clinical outcome in OS. Taken together, the method described was highly predictive for known OS-associated genes, particularly genes with low mutation frequency. Furthermore, the present study revealed that several of the identified genes were bona fide drivers. Therefore, the present study described an avenue for the identification of driver genes from large amounts of cancer genome sequencing data.

\section{Materials and methods}

Data collection. The mutation data used in the present study was derived from COSMIC (version 79; https://cancer. sanger.ac.uk/cosmic). OS missense mutations were selected for further study. The present study focused only on those mutations that were predicted to be pathogenic (defined as cancerous or damaging) by the FATHMM-MKL algorithm. The FATHMM score ranged between 0 to 1 , and variants with a score $>0.7$ were considered to be pathogenic (11) (predicting the functional, molecular and phenotypic consequences of amino acid substitutions using hidden Markov models). The official gene names corresponding to the pathogenic mutations were obtained from the National Center for Biotechnology Information (https://www.ncbi.nlm.nih.gov/). Following the removal of duplicated genes, 882 OS-associated genes were identified. Functional gene sets (FGS) were obtained from the literature (12), and included genes involved in signaling and cancer-associated pathways and hallmarks. The Cancer Gene Census (CGC; cancer.sanger.ac.uk/census), which includes gene mutations causally implicated in cancer, was downloaded. The CGC is widely used as the gold standard to evaluate the effect of predicted driver genes (10). OS-associated genes were downloaded from the OS database (osteosarcoma-gene association database, http://osteosarcoma-db.uni-muenster.de/). The gene expression dataset (GSE42352) (13) was obtained from the Gene Expression Omnibus (GEO; www.ncbi.nlm.nih.gov/geo).
Network and influence graph construction. The network was built using the Human Protein Reference Database (HPRD, release 9; www.hprd.org). Self-interactions were deleted, and 39,240 interactions among 9,616 proteins were identified. The mutated genes and the FGS were mapped onto the network. Node $\mathrm{i}$ and $\mathrm{j}$ represent genes, and the edge represents an interaction between gene $i$ and gene $j$ in the network. The influence graph presents the influence of the mutated genes on these genes in the FGS. Using the network, a bipartite graph was generated, in which nodes on one side represented the mutated genes and nodes on the other side represented the FGS. Edges were drawn if gene $i$ and gene $j$ had an interaction according to the known gene network, i.e., the influence graph. The aim of the algorithm was to identify mutated genes that were connected to the majority of genes in the FGS. The mutated genes were ranked according to their z-score using the following equation:

$$
\mathrm{z}=\frac{d_{\mathrm{AF}}-\mu_{\mathrm{AF}}}{\sigma_{\mathrm{AF}}}
$$

Where $\mathrm{d}_{\mathrm{AF}}$ is the enrichment score of mutated genes and the FGS, $\mu_{\mathrm{AF}}$ is the expected mean of $\mathrm{d}_{\mathrm{AF}}$ and $\sigma_{\mathrm{AF}}$ is the standard deviation of $\mathrm{d}_{\mathrm{AF}}$.

First, a network was constructed using the HPRD database, and the mutated genes and the FGS were mapped to the network. Second, the association between the mutated genes and the FGS was measured using a bipartite graph. In the bipartite graph, the nodes on the left represented the mutated genes, while the nodes on the right represented the FGS. An edge was drawn if nodes on each side interacted in the network. The number of edges for each of the mutated genes was subsequently calculated. Finally, the z-score was used as the driver gene score. A mutated gene with a z-score $>2$ was considered a driver gene (14).

Performance benchmarking. The well-studied CGC dataset was used as an approximate benchmarking dataset, as standard benchmarking is impractical due to lack of ground truth (14). The developed method was compared with MUFFINN (www.inetbio.org/muffinn/search.php), which is a method for prioritizing cancer genes that accounts for not only mutations in individual genes but also those in neighboring genes connected in functional networks. Candidate cancer genes were identified by NDmax on HumanNet V1 (http://www. functionalnet.org/humannet/about.html). Precision, recall and F1 scores were based on the top 100 genes in our study and were calculated as follows.

$$
\begin{gathered}
\text { Pr ecision }=\frac{(\# \text { Mutated genes in CGC }) \cap(\# \text { Genes found in our method })}{(\# \text { Genes found in our method })} \\
\operatorname{Re} \text { call }=\frac{(\# \text { Mutated genes in CGC }) \cap(\# \text { Genes found in our method })}{(\# \text { Mutated genes in CGC })} \\
F 1 \text { Score }=2 \times \frac{\text { Pr ecision } \times \text { Recall }}{\text { Precision }+ \text { Recall }}
\end{gathered}
$$

Genes found in our method refers to genes identified using the proposed method.

Identification of candidate OS driver genes as putative module biomarkers. The identified OS driver genes were validated as putative module biomarkers based on their ability to 
distinguish between OS and control samples using the random forest method (15). The performance of the classification model was assessed using receiver operating characteristic (ROC) curves and the area under the curve (AUC) (16).

GO and pathway enrichment analysis. To interpret the biological significance of the OS driver genes, Gene Ontology (GO; http://geneontology.org/) and Kyoto Encyclopedia of Genes and Genomes (https://www.genome.jp/kegg/) pathway enrichment analyses were performed using the online tool Database for Annotation, Visualization and Integrated Discovery (DAVID; version 6.7; https://david.ncifcrf.gov/). Enrichment analysis was calculated using the hypergeometric test. Only terms with adjusted $\mathrm{P}<0.05$ were considered.

Subnetwork generation. To better understand the interaction between the OS driver genes, a subnetwork consisting of the OS driver genes was generated using GenRev software V1 (17). The interaction network was sourced from the Pathway Commons database (Release 1), which is built on publicly available pathway data. The GenRev algorithm requires two inputs, network information and a set of input genes (termed seed genes), to calculate a subnetwork containing the seed gene and non-seed genes (linker genes). The present study used the limited k-walk algorithm (18), with $\mathrm{k}=3$, to evaluate the relevance of seed genes in relation to linker genes by using random walk algorithm.

\section{Results}

Identification of OS driver genes. Based on the FATHMM score of each variant, variants were labeled as pathogenic or neutral (11). As the non-pathogenic variants predicted by FATHMM are not likely to be implicated in cancer, the present study focused solely on the pathogenic variants, similarly to previously published studies $(7,19)$. Furthermore, this approach reduces the noise of false positive somatic mutations. A total of 1,244 pathogenic mutations in 882 genes were identified. The genes harboring pathogenic variants were ranked using a computational approach as shown in Fig. 1. A mutated gene with a $\mathrm{z}$-score $>2$ was considered a driver gene (14). Using this approach, a total of 15 driver genes were identified. The results form Table I demonstrates that tumor protein $\mathrm{p} 53$ (TP53) ranked first out of the driver genes.

Integrating protein interactions improves the enrichment of $O S$ genes. In order to evaluate the ability of the approach developed in the present study to detect driver genes, the results were compared with results obtained using the MUFFINN algorithm. The MUFFINN online server requires a set of genes and mutation frequencies as input. The algorithm takes into account somatic mutations both in genes and their neighbors connected in functional networks (10). MUFFINN can also detect mutations in indirect neighbor genes by diffusing the mutation occurrence information throughout the network. The output is a list of ranked cancer genes (10). Based on the MUFFINN score, genes were arranged in descending order. Precision, recall and F1 scores were based on the top $\mathrm{N}$ genes $(20,21)$. In the present study, the predictive performances for the top 100 candidates were comparable. The performance

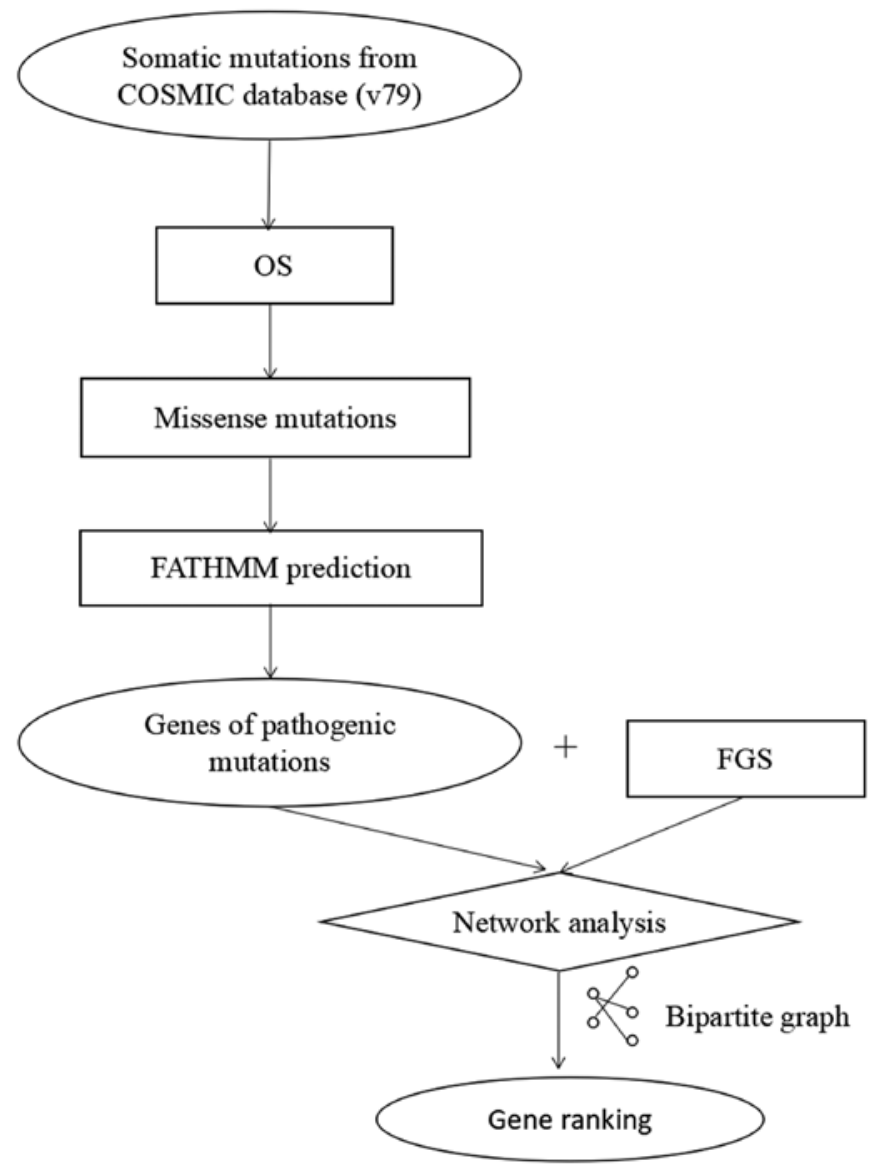

Figure 1. Illustration of OS driver gene identification. COSMIC (version 79) data was used. OS was selected as the cancer type and the FATHMM algorithm was used to retrieve the pathogenic OS missense mutations. The bipartite graph reflects the effects of the mutated genes on the FGS COSMIC, Catalogue of Somatic Mutations in Cancer; OS, osteosarcoma; FATHMM, Functional Analysis Through Hidden Markov Models; FGS, functional gene sets.

of the method developed in the present study and MUFFINN were evaluated, and the former exhibited significant improvement by using mutational data from direct neighbors in the network. As displayed in Fig. 2, the precision, recall and F1 score curves for the top 30 genes obtained using the method developed in the present study are higher than the curves obtained using MUFFINN. However, the scores for the genes after the top 30 genes were higher using MUFFINN.

It is worth noting that, TP53 (a well-known cancer gene) (22) was ranked first in both the method developed in the present study and MUFFINN. MUFFINN revealed that UBE2I (ubiquitin conjugating enzyme E2 I) ranked second. UBE2I is not a mutated gene, but can be connected to the mutated genes. Overall, the method developed in the present study performed better than MUFFINN with respect to the CGC, particularly for the top 30 genes.

Confirmation of predicted OS driver genes. The OS gene database and the CGC were used to investigate whether the predicted genes had been previously reported. Among the 15 identified OS driver genes, 13 genes overlapped with the OS gene database or the CGC (Fig. 3). Statistical analysis was performed in order to determine whether the overlapped genes 
Table I. Genes ranked by the driver gene score developed in the present study.

\begin{tabular}{lccc}
\hline Gene & Score & $\begin{array}{c}\text { Which exist } \\
\text { in CGC? }\end{array}$ & $\begin{array}{c}\text { Which exist in OS } \\
\text { gene database? }\end{array}$ \\
\hline TP53 & 10.29 & Yes & Yes \\
EGFR & 8.08 & Yes & Yes \\
CREBBP & 7.19 & Yes & Yes \\
SMAD4 & 5.87 & Yes & Yes \\
RB1 & 5.05 & Yes & Yes \\
PTK2 & 4.76 & No & Yes \\
TRAF6 & 4.61 & No & Yes \\
SYK & 4.39 & Yes & No \\
PAK1 & 3.21 & No & No \\
RASA1 & 3.21 & No & No \\
FN1 & 3.06 & No & Yes \\
VIM & 2.99 & No & Yes \\
KDR & 2.47 & Yes & Yes \\
LRP1 & 2.33 & No & Yes \\
SOCS1 & 2.03 & Yes & No \\
\hline
\end{tabular}

were randomly obtained from the 882 pathogenic genes. The P-value from the hyper-geometric test was $1.707 \times 10^{-13}$, which demonstrated that the identified OS driver genes were not randomly obtained. The results indicated that the approach developed in the present study detected 15 driver genes that are highly associated with OS.

Genes in the disease-associated network. To further explore the biological significance of the 15 OS driver genes, the driver genes were mapped to the interaction network. The 15 driver genes were inputted into the GenRev software, and 15 seed genes and their neighbors were mined. The interaction of the 15 OS genes is presented in Fig. 4, which demonstrates that all 15 OS drivers were connected in a subnetwork, where purple and red vertices represent the linker and seed genes, respectively. The subnetwork included 39 genes (15 seed genes and 24 linker genes) and 49 edges (Fig. 4). Among the 24 linker genes, 10 linker genes overlapped with genes in the OS gene database; however, the association between the other 14 genes and OS is unclear.

Functional analysis. To further investigate the biological function of the 15 driver genes, functional enrichment analysis was performed using DAVID. A number of the predicted driver genes were significantly enriched in biological functions related to tumorigenesis, including 'regulation of signal transduction' and 'regulation of cell communication' (Fig. 5A).

Validation of potential OS driver genes. To further validate the predicted OS driver genes, these genes were used to distinguish cancer samples from controls. The gene expression dataset GSE42352 was obtained from the GEO, and consisted of 15 controls and 103 OS samples. Moreover, the results were compared with the results obtained using 13 biomarkers collected from a previous study (23). The performance of the 15 predicted OS driver genes and the 13 biomarkers was evaluated using a random forest classifier and 5-fold cross-validation. The ROC curves and AUC values for the classifications of the 15 predicted OS driver genes and the 13 biomarkers are shown in Fig. 5B. The AUC was 1 for the 15 predicted OS driver genes and 0.97 for the 13 known biomarkers. This result revealed that the identified driver genes performed well compared with the known biomarkers, which demonstrated that the 15 OS driver genes are related to OS.

\section{Discussion}

Owing to the development of next-generation sequencing, genomic sequencing is a new paradigm in disease research (24). A number of somatic mutations in cancer have been reported from sequencing data (5). As only a limited number of mutations are drivers, it is critical to screen driver mutations from passenger mutations (5). Since the somatic mutations in the COSMIC database were identified by genomic sequencing, some false positive somatic mutations exist, as the early methods for genome/exome sequencing somatic mutations were less reliable than the new method (25). Although multiple computational methods have been used to predict the pathogenicity of mutations, their utility is limited (5). The present study presented an approach for integrating mutation data and networks to identify OS driver genes. FATHMM is a tool combined with other tools to predict driver genes (9). However, the top ranked genes often receive more attention and are more important than the lower ranked genes $(10,14,21)$.

The results revealed that the method was effective in detecting driver genes. A total of 15 driver genes were identified in the present study, of which 13 have been reported previously (11 genes in the OS gene database and 8 genes in the CGC). Based on a literature search, among these identified genes in our study, TP53 mutations are one of the most common genetic aberrations in OS. Evidence suggests that EGFR is implicated in the development and progression of OS (26). A meta-analysis revealed that TP53 is an effective biomarker of survival time in patients with OS (27). Epidermal growth factor receptor (EGFR) belongs to the protein kinase superfamily. EGFR mutations enhance the kinase activity of EGFR, which activates pro-survival pathways, including RAS/MAPK pathway (28). Evidence suggests that EGFR is implicated in the development and progression of OS (26). CREBBP (CREB binding protein) plays a central role in transcriptional activation. SMAD4 (SMAD family member 4) encodes a protein that is a part of the transforming growth factor $\beta$ (TGF- $\beta$ ) pathway, which has been implicated in cancer, including OS (29). RB1 (RB transcriptional corepressor 1) is a tumor suppressor gene, of which mutations are positively correlated with the survival rate of patients with OS. PTK2 (protein tyrosine kinase 2) encodes a cytoplasmic protein tyrosine kinase, which drives tumor growth through its pro-proliferative and antiapoptotic functions (30). TRAF6 (TNF receptor associated factor 6) is an oncogene that plays a crucial role in RAS-mediated oncogenesis in lung cancer (31). A previous study reported that the overexpression of TRAF6 is correlated with the invasion of OS cells (32). SYK serves a dual role as a tumor 

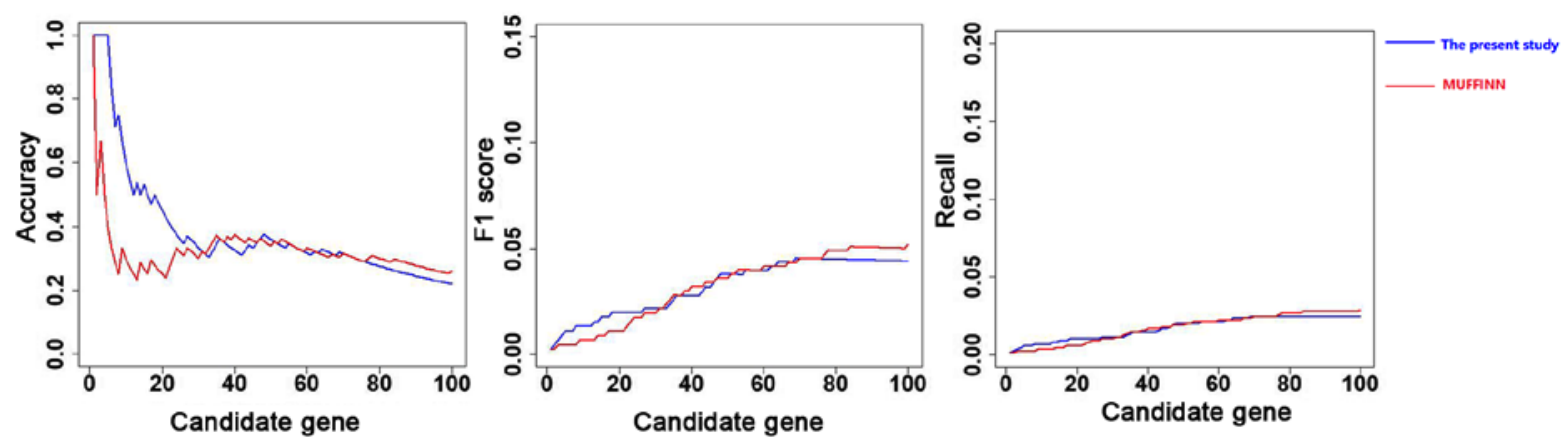

Figure 2. Comparison of the precision, recall and F1 scores for the top-ranking genes using the method developed in the present study and MUFFINN. The $\mathrm{x}$-axis represents the number of top-ranking genes involved in the precision, recall and F1 score calculation. The y-axis represents the score of the given metric. MUFFINN, Mutations For Functional Impact on Network Neighbors.
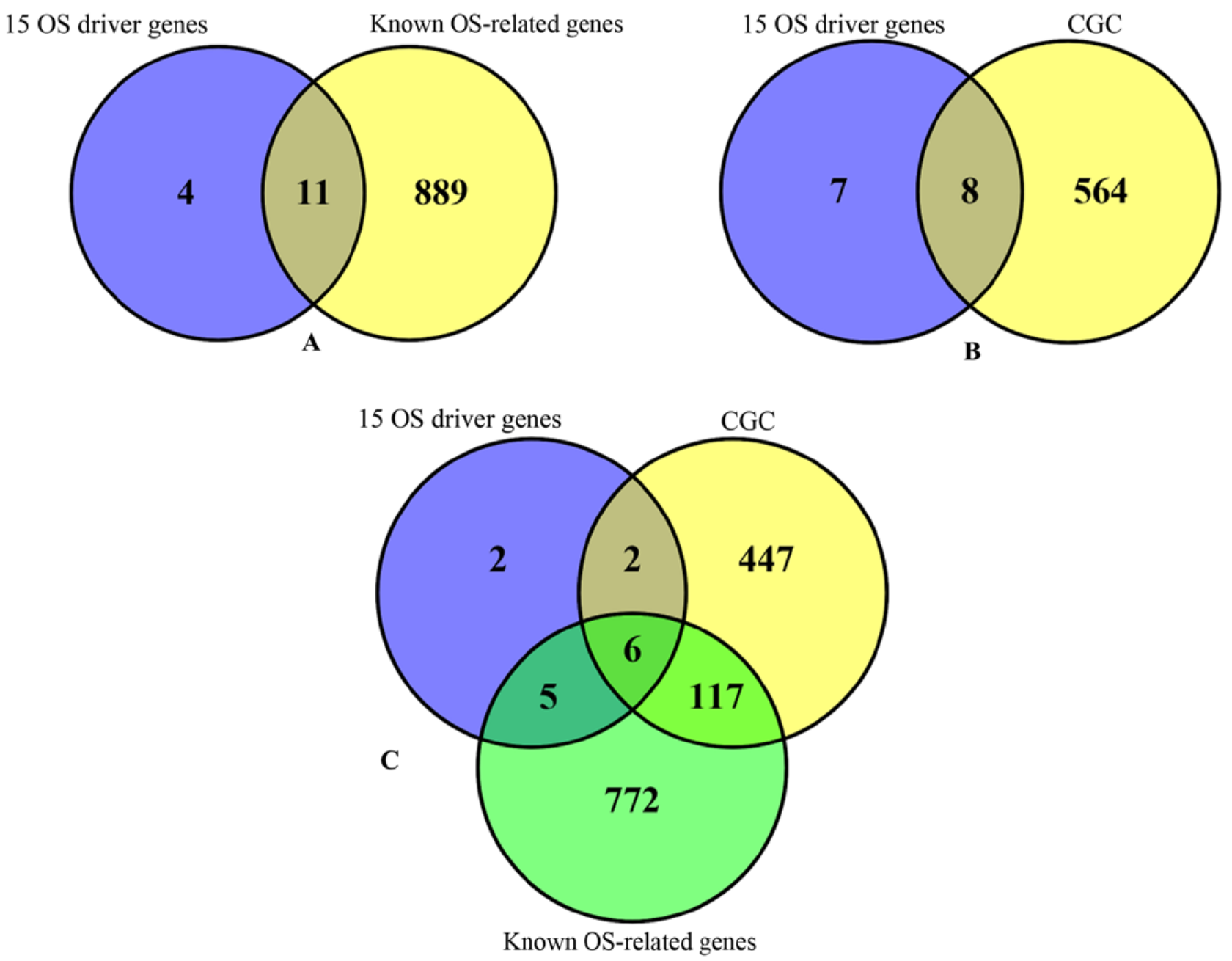

Figure 3. OS-related genes and known cancer genes. (A) A total of 900 known OS-related genes were obtained from the OS database, 11 of which were identified by the method developed in the present study. (B) A total of 572 CGC genes were obtained from COSMIC, 8 of which were identified by the method developed in the present study. (C) OS-related genes and known cancer genes obtained from COSMIC. OS, osteosarcoma; CGC, Cancer Gene Census; COSMIC, Catalogue of Somatic Mutations in Cancer.

promoter in certain tumors, including B-cell lymphocytic leukemia, pancreatic cancer and lung cancer), and as a tumor suppressor in other types of cancer, including breast cancer and melanoma (33). A previous study suggested that SYK may be associated with OS (34). PAK1 [p21 (RAC1) activated kinase 1] is a kinase that confers chemoresistance and poor outcome in non-small cell lung cancer (35). RASA1 (RAS p21 protein activator 1) acts as a tumor suppressor gene that is frequently inactivated in various types of cancer, including hepatocellular carcinoma (36). Compared with normal human osteoblasts, FN1 downregulation has been reported in human osteosarcoma cell lines (37). In addition, a random forest 


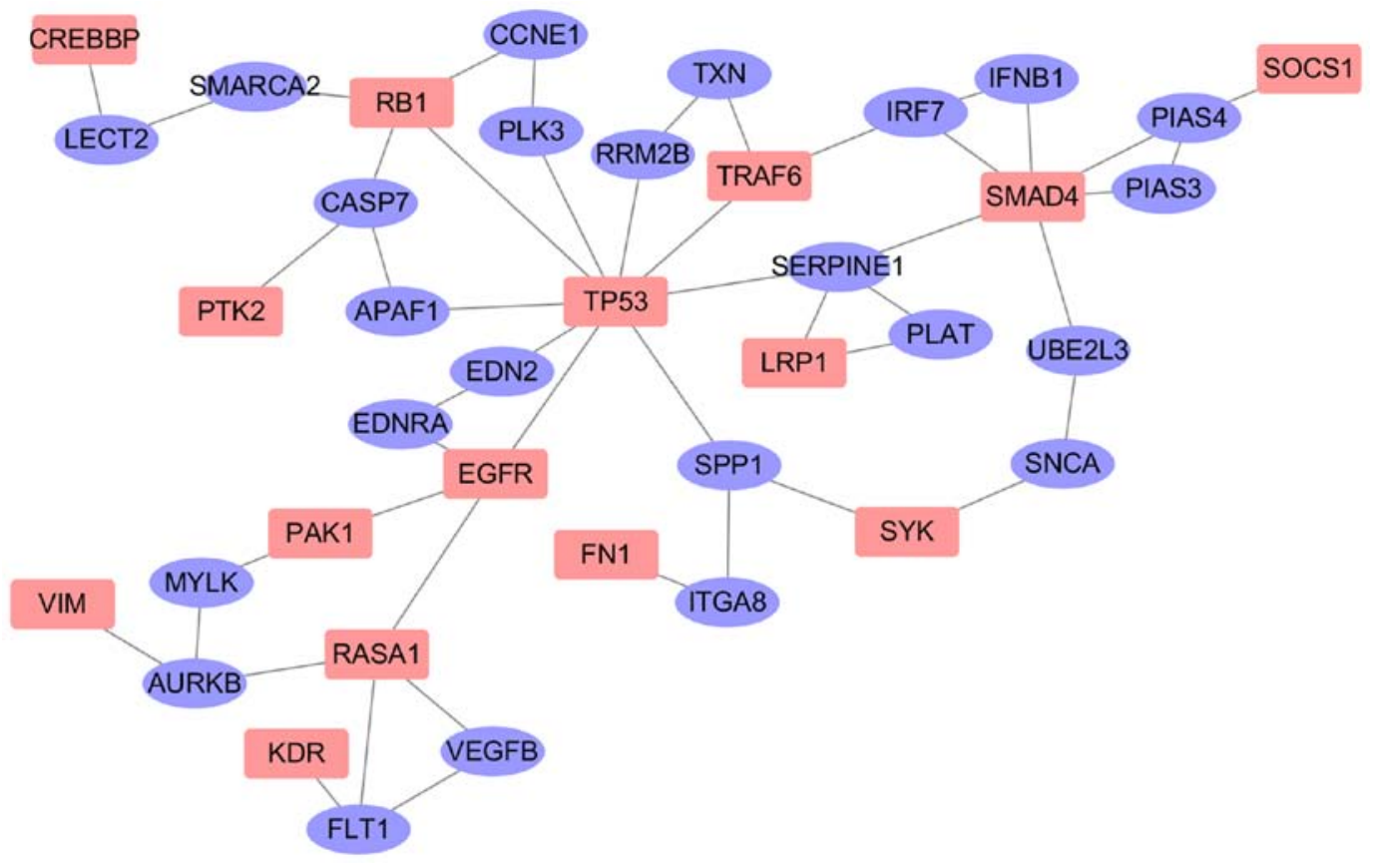

Figure 4. Interaction network with the predicted osteosarcoma driver genes. The interactions among the 15 genes were obtained from the Human Protein Reference Database. The red nodes represent the 15 identified genes, whereas the purple nodes represent the neighboring genes.

A

The GO enrichment results of top 15 driver genes
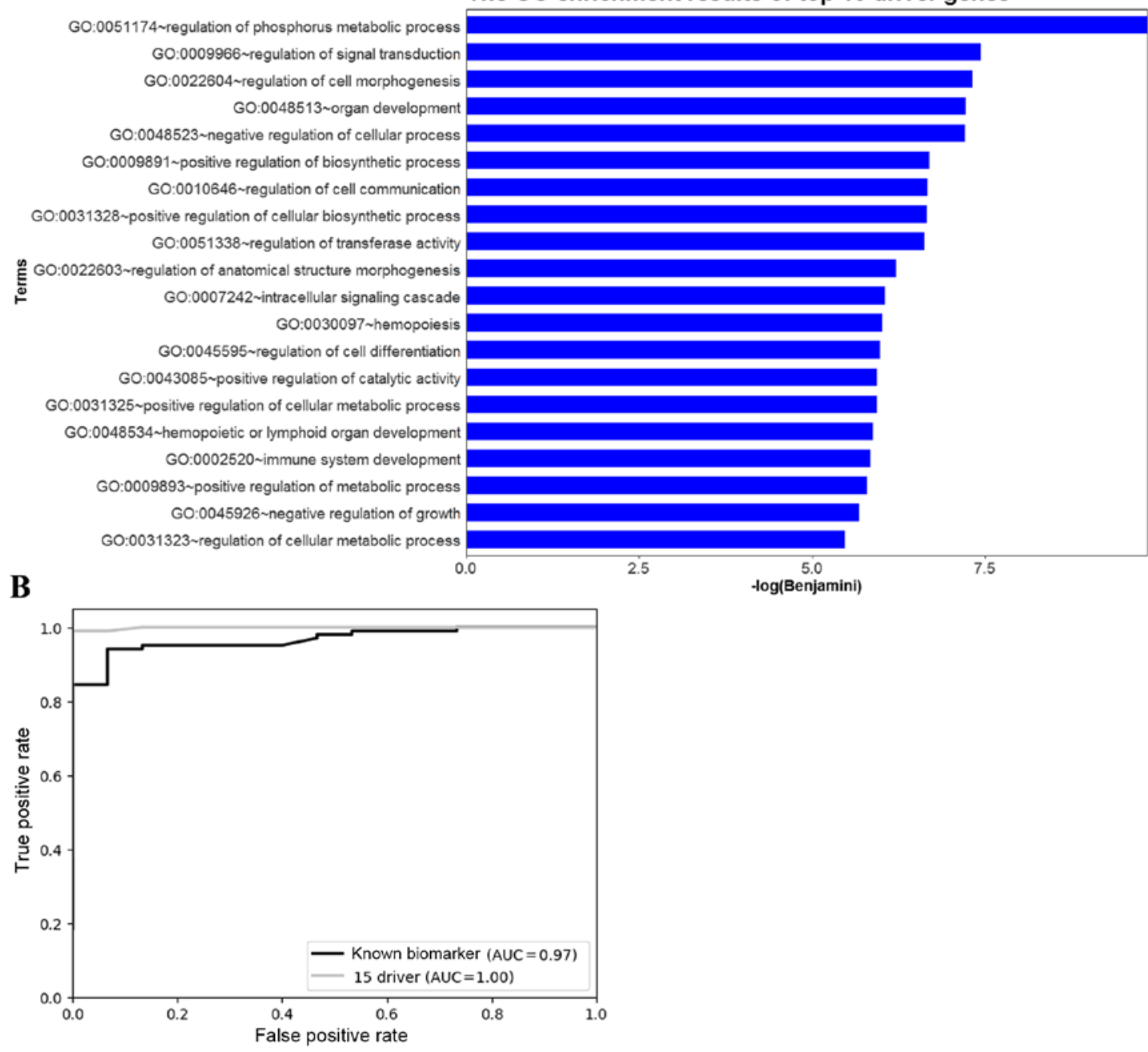

Figure 5. GO enrichment and ROC curves. (A) The GO enriched terms ( $\mathrm{P}<0.05)$ of the 15 OS driver genes identified in the present study. (B) ROC curves obtained using the driver genes and published biomarkers (24). GO, Gene Ontology; ROC, receiver operating characteristic; AUC, area under the curve. 
classifier was used to demonstrate the ability of the predicted drivers to distinguish between OS and control samples, and the AUC values suggested a good classification performance. The 15 driver genes outperformed the known biomarkers of OS, suggesting that the predicted driver genes are related to OS.

The present study had a number of limitations. Experimental validation using small interfering RNA and cell viability assays was not performed. Therefore, future investigations are required to further validate the potential driver genes. Furthermore, despite the good performance for detecting OS driver genes, the model has certain shortcomings. Firstly, the network information is incomplete, and genes that could not be mapped to the network were filtered out. Secondly, only the missense mutations were explored, and other types of mutations require further investigation as, for example, synonymous mutations have been reported to play a crucial role in cancer risk (38). Hence, the predictive power of the approach developed in the present study may be enhanced by additional functional network information.

Taken together, the present study developed a practical approach to mine COSMIC for potential OS driver genes. This approach may be generalized to identify new diagnostic biomarkers and therapeutic targets for OS. Additionally, although only OS-related genes were explored in the present study, the method is broadly applicable to other cancer types available in COSMIC.

Inferring the driver genes in cancer is one of the goals of systems biology. Given that COSMIC provides a significant amount of mutation data, the optimization of the use of these data to identify the driver genes in a given cancer type is important. In the present study, known interactions were used to consider the effect of mutated genes on a set of functional genes, and 15 OS driver genes were identified. These genes were functionally enriched in OS-associated biological functions, indicating that these genes are involved in OS. Furthermore, the method developed in the present study outperformed the MUFFINN algorithm. Therefore, the network strategy of prioritizing OS genes described in the present study is effective.

\section{Acknowledgements}

Not applicable.

\section{Funding}

No funding was received.

\section{Availability of data and materials}

The datasets generated and/or analyzed during the current study are available from COSMIC (https://cancer.sanger. ac.uk/cosmic).

\section{Authors' contributions}

$\mathrm{ZS}$ and $\mathrm{KH}$ conceived the experiment design. ZS performed the data analysis. ZS wrote the manuscript. ZS and KH revised the manuscript. All authors have read and approved the final version of the manuscript.

\section{Ethics approval and consent to participate}

Not applicable.

\section{Patients consent for publication}

Not applicable.

\section{Competing interests}

The authors declare that they have no competing interests.

\section{References}

1. Ritter J and Bielack SS: Osteosarcoma. Ann Oncol 21 (Suppl 7): vii320-vii325, 2010.

2. Tumijan W, Khattak MN, Nadiah WA and Naing NN: Five-year survival of osteosarcoma patients in hospital universiti sains malaysia (Husm): An eleven year review. Res J Pharmacy Technol 11: 3534-3542, 2018

3. Chen X, Bahrami A, Pappo A, Easton J, Dalton J, Hedlund E, Ellison D, Shurtleff S, Wu G, Wei L, et al: Recurrent somatic structural variations contribute to tumorigenesis in pediatric osteosarcoma. Cell Rep 7: 104-112, 2014.

4. Martincorena I, Raine KM, Gerstung M, Dawson KJ, Haase K, Van Loo P, Davies H, Stratton MR and Campbell PJ: Universal patterns of selection in cancer and somatic tissues. Cell 171: 1029-1041.e21, 2017.

5. Cheng F, Zhao J and Zhao Z: Advances in computational approaches for prioritizing driver mutations and significantly mutated genes in cancer genomes. Brief Bioinform 17: 642-656, 2016.

6. Forbes SA, Beare D, Gunasekaran P, Leung K, Bindal N, Boutselakis H, Ding M, Bamford S, Cole C, Ward S, et al: COSMIC: Exploring the world's knowledge of somatic mutations in human cancer. Nucleic Acids Res 43 (Database Issue): D805-D811, 2015.

7. Rogers MF, Shihab HA, Gaunt TR and Campbell C: CScape: A tool for predicting oncogenic single-point mutations in the cancer genome. Sci Rep 7: 11597, 2017.

8. Shihab HA, Rogers MF, Gough J, Mort M, Cooper DN, Day IN, Gaunt TR and Campbell C: An integrative approach to predicting the functional effects of non-coding and coding sequence variation. Bioinformatics 31: 1536-1543, 2015.

9. Dong C, Guo Y, Yang H, He Z, Liu X and Wang K: iCAGES: Integrated CAncer GEnome Score for comprehensively prioritizing driver genes in personal cancer genomes. Genome Med 8: $135,2016$.

10. Cho A, Shim JE, Kim E, Supek F, Lehner B and Lee I: MUFFINN: Cancer gene discovery via network analysis of somatic mutation data. Genome Biol 17: 129, 2016.

11. Shihab HA, Gough J, Cooper DN, Stenson PD, Barker GL, Edwards KJ, Day IN and Gaunt TR: Predicting the functional, molecular, and phenotypic consequences of amino acid substitutions using hidden Markov models. Hum Mutat 34: 57-65, 2013.

12. Merid SK, Goranskaya D and Alexeyenko A: Distinguishing between driver and passenger mutations in individual cancer genomes by network enrichment analysis. BMC Bioinformatics 15: 308, 2014.

13. Kuijjer ML, Peterse EF, van den Akker BE, Briaire-de Bruijn IH, Serra M, Meza-Zepeda LA, Myklebost O, Hassan AB, Hogendoorn PC and Cleton-Jansen AM: IR/IGF1R signaling as potential target for treatment of high-grade osteosarcoma. BMC Cancer 13: 245, 2013.

14. Zhang $\mathrm{T}$ and Zhang $\mathrm{D}$ : Integrating omics data and protein interaction networks to prioritize driver genes in cancer. Oncotarget 8: 58050-58060, 2017.

15. Breiman L: Random forests. Machine Learning 45: 5-32, 2001.

16. Wen Z, Liu ZP, Liu Z, Zhang Y and Chen L: An integrated approach to identify causal network modules of complex diseases with application to colorectal cancer. J Am Med Inform Assoc 20: 659-667, 2013.

17. Zheng $\mathrm{S}$ and Zhao Z: GenRev: Exploring functional relevance of genes in molecular networks. Genomics 99: 183-188, 2012. 
18. Dupont P, Callut J, Dooms G, Monette JN and Deville Y: Relevant subgraph extraction from random walks in a graph. Research Report UCL/FSA/INGI RR 2006-07, 2006.

19. Miao YR, Liu W, Zhang Q and Guo AY: lncRNASNP2: An updated database of functional SNPs and mutations in human and mouse lncRNAs. Nucleic Acids Res 46: D276-D280, 2018.

20. Bashashati A, Haffari G, Ding J, Ha G, Lui K, Rosner J, Huntsman DG, Caldas C, Aparicio SA and Shah SP: DriverNet: Uncovering the impact of somatic driver mutations on transcriptional networks in cancer. Genome Biol 13: R124, 2012.

21. Hou JP and Ma J: DawnRank: Discovering personalized driver genes in cancer. Genome Med 6: 56, 2014.

22. Liu Y, Zhang X, Han C, Wan G, Huang X, Ivan C, Jiang D, Rodriguez-Aguayo C, Lopez-Berestein G, Rao PH, et al: TP53 loss creates therapeutic vulnerability in colorectal cancer. Nature 520: 697-701, 2015.

23. Li G, Zhang W, Zeng H, Chen L, Wang W, Liu J, Zhang Z and Cai Z: An integrative multi-platform analysis for discovering biomarkers of osteosarcoma. BMC Cancer 9: 150, 2009.

24. Behjati S and Tarpey PS: What is next generation sequencing. Arch Dis Child Educ Pract Ed 98: 236-238, 2013.

25. Ma M, Wang C, Glicksberg BS, Schadt EE, Li SD and Chen R: Identify cancer driver genes through shared Mendelian disease pathogenic variants and cancer somatic mutations. Pac Symp Biocomput 22: 473-484, 2017.

26. Do SI, Jung WW, Kim HS and Park YK: The expression of epidermal growth factor receptor and its downstream signaling molecules in osteosarcoma. Int J Oncol 34: 797-803, 2009.

27. Fu HL, Shao L, Wang Q, Jia T, Li M and Yang DP: A systematic review of p53 as a biomarker of survival in patients with osteosarcoma. Tumour Biol 34: 3817-3821, 2013.

28. Henson E, Chen Y and Gibson S: EGFR family Members' regulation of autophagy is at a crossroads of cell survival and death in cancer. Cancers (Basel) 9: E27, 2017.

29. Lamora A, Talbot J, Mullard M, Brounais-Le Royer B, Redini F and Verrecchia F: TGF- $\beta$ signaling in bone remodeling and osteosarcoma progression. J Clin Med 5: E96, 2016.
30. Sulzmaier FJ, Jean C and Schlaepfer DD: FAK in cancer: Mechanistic findings and clinical applications. Nat Rev Cancer 14: 598-610, 2014.

31. Starczynowski DT, Lockwood WW, Deléhouzée S, Chari R, Wegrzyn J, Fuller M, Tsao MS, Lam S, Gazdar AF, Lam WL and Karsan A: TRAF6 is an amplified oncogene bridging the RAS and NF- $\kappa \mathrm{B}$ pathways in human lung cancer. J Clin Invest 121: 4095-4105, 2011.

32. Meng Q, Zheng M, Liu H, Song C, Zhang W, Yan J, Qin L and Liu X: TRAF6 regulates proliferation, apoptosis, and invasion of osteosarcoma cell. Mol Cell Biochem 371: 177-186, 2012.

33. Krisenko MO and Geahlen RL: Calling in SYK: SYK's dual role as a tumor promoter and tumor suppressor in cancer. Biochim Biophys Acta 1853: 254-263, 2015.

34. Sun L, Li J and Yan B: Gene expression profiling analysis of osteosarcoma cell lines. Mol Med Rep 12: 4266-4272, 2015.

35. Chen MJ, Wu DW, Wang YC, Chen CY and Lee H: PAK1 confers chemoresistance and poor outcome in non-small cell lung cancer via $\beta$-catenin-mediated stemness. Sci Rep 6: 34933, 2016.

36. Chen YL, Huang WC, Yao HL, Chen PM, Lin PY, Feng FY and Chu PY: Down-regulation of RASA1 is associated with poor prognosis in human hepatocellular carcinoma. Anticancer Res 37: 781-785, 2017.

37. Wolf M, El-Rifai W, Tarkkanen M, Kononen J, Serra M, Eriksen EF, Elomaa I, Kallioniemi A, Kallioniemi OP and Knuutila S: Novel findings in gene expression detected in human osteosarcoma by cDNA microarray. Cancer Genet Cytogenet 123: 128-132,2000.

38. Supek F, Miñana B, Valcárcel J, Gabaldón T and Lehner B: Synonymous mutations frequently act as driver mutations in human cancers. Cell 156: 1324-1335, 2014.

This work is licensed under a Creative Commons Attribution-NonCommercial-NoDerivatives 4.0 International (CC BY-NC-ND 4.0) License. 